\title{
Intermediality of Screens in Post-Media Assemblages
}

\author{
Charu Maithani \\ University of New South Wales (Sydney, Australia) \\ E-mail: c.maithani@unsw.edu.au
}

\begin{abstract}
In contemporary artworks of so-called post-media assemblage, screens can be argued to emphasize, interconnect and rearticulate relationships between various parts in various modalities of imagemaking and display. They can be understood to produce gesturality that maintains conditions of mediality, which is the sustenance of relations between different parts of the media ensemble. This paper is an attempt to understand screens by analysing the gesturality that they propagate and not just facilitate. For this purpose, the paper interrogates the intermediality of screens in contemporary media arts that rely on this gesturality. By closely analysing contemporary media art installations such as Solar Reserve (Tonopah, Nevada) (John Gerrard, 2014) and Shadow 3 (Shilpa Gupta, 2007), this paper elaborates a concept of intermediality as an unfixed state and describes in-betweenness as enabling an openness to continuously form, unform and deform relations with different entities, thereby producing a gestural modality.
\end{abstract}

Keywords: screens, post-media assemblage, gesturality, intermediality.

Cinema or screen studies has a long reputation for analysing questions of gaze, spectacle, spectator and affect (Mulvey 1975; Metz 1982; Gunning 1986/2006). Additionally, it has also approached screens as part of cinematic architecture (Lindsay 1915; Bruno 2014). Visual arts have analysed screens as part of installations (Reiser and Zapp 2002; Shaw and Weibel 2003; Mondloch 2010), expanded cinema and projection-based forms (Youngblood 1970; Lord and Marchessault 2007; Trodd 2011). Media studies has contemplated screens as information surfaces (Huhtamo 2012), mediators (Bolter and Grusin 2000) and dynamic interfaces (Manovich 1998). Since the infiltration of digital technology across media, screens have gathered more importance as they expand their reach. Cross-disciplinary analysis has led to recognizing the ways in which they operate variously as: window (Friedberg 2006), light (Paul 2015), interface (Laurel 1991), and are linked to physical movements 
(Verhoeff 2012). This rich literature has unveiled new perspectives on the history and contemporary workings of media through screens. W. J. T. Mitchell $(2015,234)$ analysed the duality of screens in their hiding and revealing. He argues that in cinema and in television we see on and through screens as they project and display. The multiple modalities of screens do not imply that screens are platforms, formats, and/or mediums. Instead, they show what we see and are the connection between other media such as television, computers and films, an "in-between manifestation" of medium, format and platform (Acland 2012, 168). I propose the next steps to study the scope and role of screens in the various forms of contemporary postmedia assemblages that thrive on the instability and multiplicity of connections between its parts. Post-media assemblages are based on the concept of assemblages provided by Deleuze and Guattari (1980/2017) to emphasize the arrangement of its parts, human and nonhuman. As a "non-totalizable sum" (Bennett 2010, 24), assemblages are useful to emphasize the agency of nonhuman elements, and the relationality of the parts in its emergent properties. In the context of this paper, a contemporary art installation can be considered an assemblage where the shifting capacities of the components produce new relations and encounters. The capacity of screens is of particular interest in this complex network of human bodies and nonhuman things, not only in emphasizing its engagement with other parts, but also how screens can be defined in the process.

Screens have taken on additional function than merely that of display in the post-media condition. The contemporary convergence culture (Jenkins 2006) indicates easy migration of content between media, furthermore, screens are the common constituent of image production, display and distribution. In the postmedia condition, researches indicate that screens elicit new gestures, behaviours and experiences, as different functionalities of digital screens are activated when interfacing between humans and hardware, software and hardware, and humans and software (Paul 2015). ${ }^{1}$ Keeping this in mind, screens can be argued to emphasize, interconnect and rearticulate the relationship between various parts of a media assemblage, such as the image, the technological device such as a smartphone or computer, and the user - in various modalities of image-making and display. I am proposing an interpretation of screens by the way of analysing the gesturality that they produce. What would an extrapolation of the gestural performativity of screens allow us to see in terms of relations that are formed

1 Different screens initiate a different behaviour: the cinematic screen calls for a certain modality of viewing while navigating with the help of Google maps on mobile screens elicits a different response to the screen. 
by and through screens that previous studies have not? Here, I am considering other ideas of gestures where they are not just about movements of bodies. While gestures may be vital in any interaction, gesturality is always already present in the circulation and connection between parts of an unstable media assemblage. Furthermore, I argue that this gesturality is propagated and not just facilitated by screens. I specifically interrogate the intermediality of screens that aids this gesturality of screens in contemporary media arts.

Following Giorgio Agamben's idea that gestures are "not the sphere of the end itself but rather the sphere of pure and endless mediality," $(2000,58)$ gesturality can be understood as maintaining conditions of mediality, that is the sustenance of relations between different parts of the media ensemble. However, my concern in studying this kind of meaning of gestures is to open a door to the discussion on the instability of the identity of screens and their existence in the materialization of relations and enactment of agency. Such contemplation stems from posthumanist concerns, where agency of nonhuman entities is interrogated in order to understand the organization of dynamic relations to form a structure.

\section{In the Mix - Screens, Images and Human Bodies in Post-Media}

Gestures have classically been understood as a form of communication that plays out via the movements of human bodies (Darwin 1872; Eibl-Eibesfeldt 1972), signifying meaning through their expressiveness. This understanding recognizes gestures as a means of transmitting information about feelings and ideas, however ambiguous or opaque they may be. More recently, and in relation to onscreen gestures, Michelle Langford $(2006,137)$ suggested two main types of gestures to be found in cinema. Histrionic gestures comprise codes that have developed historically to contain a specific meaning while naturalistic gestures are more impulsive and motivated towards an action. Both kinds are encoded and can be deciphered in order to produce signification. While Langford counters these classifications of gestures with examples where gestures do not have a fixed meaning, ${ }^{2}$ she nevertheless provides a semiotic understanding of gestures where these are used as a mode of communication along with or in addition to speech. Such a one-dimensional comprehension of gestures does not take into account the context of the performance of gestures and the body performing the gestures.

2 Langford (2006) analyses the works of Werner Schroeter as "gestural cinema" and the images as allegorical figures.. 
It would be a mistake to consider gestures to substitute for words or compliment language such as in the example of a nod to mean only agreement to something. In several instances, the co-mingling of gestural significations is such that consideration of one aspect renders the discussion incomplete. For example, a nod also carries a history of body movements ingrained in the sociality of a specific time and place. In some cultures, such as in Greece and Bulgaria, a nod is considered to be a refusal (LeFrance and Mayo 1978). However, my point is not about whether an action or movement can be decoded, but rather that gestures are already multivalent. In the field of social robotics, for example, where specific kinds of gestures are studied as being part of human behaviour, ironically, the multivalency of the very same human gestures is ignored.

Moreover, the location of gestures in human body gives an incomplete understanding of the relations between humans and nonhuman entities in such complex interwoven relations as we have, for example, in cinema. Lesley Stern $(2008,206,188)$ interrogates a gestural discourse that is set up in cinema with the relations between the actors, director, cameraman, editor and the instruments used by them. Bringing together the concepts of mimesis and pathos, she argues that gestures are mimetic as each gesture calls for a gestural response (Stern 2008, 202). In cinema, the gestures of the actors elicit a gestural response from the spectators as well, which does not mean the repetition of the actions of the actors, but a transfer of the intensity of the gesture in the spectator's bodies affectively. Mimesis is in the intensity of the gesture and not in its meaning (Stern 2008, 202). The gestures of the film camera, editing or sound have the effect of moving the viewers. Akira Mizuta Lippit $(2008,117)$ broadly identifies two gesturing bodies with different approaches to "inscribe" gestures: that of the actors and various cinematic apparatuses such as camera (that gesture by panning, tilting, point of view shots) and editing (gesturing a space-time continuity or discontinuity, jarring movements or continuous movements by consecutive shots). Lippit coins the term "digesture" to talk about the gestures of these two bodies. In various works of experimental cinema, Lippit $(2008,121)$ describes the gestures of these two bodies that could give rise to new gestures. For example, in Martin Arnold's works, pièce touchée (1989), Passage a l'acte (1993) and Alone. Life Wastes Andy Hardy (1998), movement of actors from Hollywood film scenes are de- and re-constructed. Arnold repeats the movements of actors over and over again, glitching, slowing, reversing and inverting them. The gestures of the actor's body and the editing apparatus give rise to new gestures that are not situated in the original work. Further on, Lippit describes the work Deanimated (The 
Invisible Ghost, 2002) by Martin Arnold as an instance where the erasure of the bodies takes place digitally. Arnold edits out the actors or their mouths leading to gestures without bodies (Lippit 2008, 127). Similar to Stern, Lippit too recognizes the agency of other non-human actors in cinema such as the camera and editing. On the one hand, Stern's observations are on the gestural dialogues between various bodies and the translation of these into affect - an emotional experience for the viewers. On the other hand, Lippit is firmly concerned with the effect of gestures on the bodies and their erasure. However, both are concerned with the "migration" of gestures between bodies, cultures and contexts (Noland 2008, xvi). Moreover, along with the movement of gestures there are also concerns regarding their inscription. There is always someone or something, a human or nonhuman entity, witnessing, recording, effected or affected by the gestures.

In this unravelling of gestural migration described by Stern and Lippit, the location and meaning of gestures has been complicated. In terms of the location of the gesture, in Stern and Lippit's discussions, gestures have been separated from the context of human body in order to interrogate the gestures of non-human bodies. Stern moves away from the meaning of gestures to the intensity of gestures, in other words, the affect produced by the gesture; and Lippit is not interested in the meaning but in the gesturing body and the lack of a body. However, their discussion is limited to cinema and the cinematic instruments. Here, the role of spectator is also limited to simply being affected upon. It does not consider the networks of relations of various entities with dynamic capacities in a post-media assemblage. In such a configuration, the gestural discourse extends to the network of humans, screens, images and the technological apparatus. There are not just two gesturing bodies as argued by Lippit but several other bodies contributing to the gestural conversation.

Further on, nonhuman entities such as images and screens are endowed with greater mobility where both can traverse various media forms with ease, taking on new capacities and building new connections. An example of such a transversal movement and reconnection of entities can be seen in changing the orientation of a mobile phone from vertical to horizontal, where the image too changes its orientation. Here, the movement of the beholder's hands almost simultaneously changes the orientation of the screen and image. A number of entities are gesturing when the orientation of a phone changes: the accelerometer (inside the mobile phone) prompts the change in the orientation of the image, the image on the screen, which actually rotates, the arm that flicks the phone in this case, the support frame of the screens that becomes visible in the gesturing, as well as 
the body of the viewer in which an affective gesturing takes place. It can also be argued that the image is gesturing to reveal itself in the change of orientation. So, even a modest action of changing the direction of the mobile phone is a cryptic indication of which entity is gesturing - the phone, image, screen or the person. More likely, in this entanglement, gestures are not produced by one entity. Hence, it is more useful to analyse gesturality or gestural performance in the movement and interaction of different entities.

Whilst the connected but blurred boundaries of entities provide an insight into the gestural performance of the assemblage, focussing on the participation of screens illustrates its distributive performance. Some gestures are conflated by the screens in the configuration considered above. The screens connect to various bodies in their multiple modalities such as displaying graphics to the person holding the phone; and depending on the input by the person, the screens initiate the software to run a particular function. In this way, the multifaceted modalities of screens not only help in negotiating the distributed entities (of all kinds) across media but help perform this distribution. This arrangement of bodies through the reworking of connections becomes central to a new kind of gesturality that we might ascribe to nonhuman entities such as screens. Gesturality may already be present, but screens are setting up a potentiality for the gestures to circulate. Being in-between the body and the image, the body and the machine, and the image and the software, the screens interface between these multiple entities and in the process facilitate a particular kind of gesturality. They set up a space for the gestural performance of various entities which they are very much a part of. The gestural capacity of screens to create, break or expand the relations with various elements reinforces their position in a techno-social set-up by indicating that the role of screens goes beyond that of being mere containers for images. Furthermore, these gestures are not just actions or set of movements, they indicate the support and possibilities through which several components can associate and converse with each other. To elaborate on this understanding of gestures as sustaining actions, let's turn to Agamben.

In his seminal text, Notes on Gesture, Agamben (2000, 50-51) refrains from attributing two kinds of meanings that gestures are usually associated with. Firstly, they provide psychological insights; and secondly, gestures carry a symbolic meaning as usually seen in dance. Instead, he elaborates on the third meaning of gestures, placing them in the realm of non-representation (Agamben 2000, 58). By stating that gestures offer "pure and endless mediality," Agamben $(2000,58)$ refers to their betweenness. Drawing upon Aristotle's poiesis (making), which is means to an end, and praxis (action), where the action itself is the end, 
Agamben $(2000,57)$ situates gestures in the middle of poiesis and praxis, where they become means without end. He argues that both a pornographic film and a mime performance have gestures that are "suspended in and by their own mediality," as reaching the goal in both these cases would complete the act (Agamben 2000, 57). These gestures do not start an action with a purpose, nor is a particular end intended. If gestures are means that do not arrive at a certain goal, then they "endure and support" the action (Agamben 2000, 56). This means that gestures can be understood as movements that carry on the action, propelling as well as nurturing it without completing it.

Agamben (1991) has been largely inspired by Kommerall's ideas on gesture, according to which gestures are the "communication of a communicability," which refers more to the potential than an expression of certainty. Yet, gestures cannot be expressed in language (Agamben 2000, 59); not since gestures and language are two different modes of communication, but because language is incapable of communicating gestures. This is why Agamben (2000) has even referred to gestures as being a "gag," where the person cannot speak and mimes.

If gestures produce mediality, then we should examine what is understood by mediality in the context of this paper. In a reassessment of media experience in the USA since 9/11, Grusin $(2010,72)$ describes mediality as highlighting the "ways in which they [media] function as agents" and formulates a relationship between affect and mediality (see Grusin 2010, 79-80). ${ }^{3}$ While he is interested in how mediality is used to affect power, I am more interested in understanding the basis of the connection between affect and mediality. A mediality that is affective indicates a deep engagement with the qualities of the medium.

As discussed above, gesturality can be understood as sustenance of mediality, relations that sustain the gestures in certain situations. These relations are continuously negotiated as capacities. The connections not only produce movements that we are able to see, but are also "excavate(ing) inward movements that endure at a deeper level” (Pisters 2015). However, when thinking about screens, the intermediality of screens has to be acknowledged. This mediality is a betweenness of screens where they are able to articulate relations between various entities. This betweenness does not only refer to the position of screens and its intermedial travels both within and across media, but also to the modes of reconfigurations, facilitated by screens, between various entities. In order to understand the performative operations of intermediality of screens, I will consider two ways of understanding it.

3 He relies on Brian Massumi's (2002) formulation of the operation of modulating affect. 


\section{Intermedial Gesturality}

\section{a) Intermediality through movement within and across media}

In the post-media age, scholars, artists and media practitioners have become attentive to "configurations which have to do with a crossing of borders between media” (Rajewsky 2005, 46; Pethő 2014; Balme 2004, 7), interaction of materiality between media and transformation of media (Paech 2014; Spielmann 2001). Given that the interwoven medial relations in the post-media condition are relational, unstable and constantly reshaping, the conditions of intermediality also have to be redefined. Therefore, instead of approaching intermediality via the concept of media as done by Clüver (2007), Müller (2010), Paech (2014) and Elleström (2010), I find it more helpful in the contemporary media space to study intermediality as a space for transformation and emergence.

Yvonne Spielmann $(2001,61)$ insists on a fusion of elements in intermedia, in other words, where elements of the media combined cannot be recognized. She suggests understanding intermedia as a concept which can be used to address the metamorphosis between media, the construction of this metamorphosis or as a tool for aesthetic changes. Irina Rajewsky (2005, 47-48) has argued that intermediality can be understood as a "fundamental condition or category" or "a critical category for concrete analysis of specific conditions." Both Spielmann (2001) and Rajewsky (2005) seem to suggest that intermediality can be argued to denote an unfixed state. Higgins $(1981,53)$, while cautiously suggesting that intermedia is not a movement or a special quality of the artwork, declares intermediality to be a way to understand and talk about the art work. This way, intermediality can be argued to be situated in a practice and not an area of study. It can be understood as a condition and not a category of media (Elleström 2010, 39). As Jill Bennet $(2007,434)$ remarks, intermediality is "not just an issue of medium," but becomes a "transdisciplinary sphere of operation" that lies at a juncture of different practices and technologies. This juncture can be the inbetweenness described by Ágnes Pethő (2014, 474-475), where the intermediality is in a state of "becoming" - a term elaborated by Deleuze and Guattari (2004) to address a state of unfolding, instability and immanence. Intermediality then, becomes a state of potential that exists at the "molecular" level.

Understanding intermediality as a practice that moves across disciplines and media is helpful to understand the medial interconnections of screens. On the other hand, screens are employed across stages of production and display 
in various media. This way screens are not only inter-medial but also intramedial, that is they are not only between media but also within a medium as well. Interestingly, many times the inter- and intra-mediality persist together. By the way of analysing John Gerrard's work, I exemplify a gestural performance of screens through their inter- and intra-mediality as they traverse through various media. John Gerrard creates works that are real-time computer simulations of actual places and events. Gerard uses various media technologies at each step of the production of his works, starting with photographs that provide the basis for $3 \mathrm{D}$ renderings of the place. These are created first on imaging software like Maya and Photoshop, and then on the gaming engine, Unigine. The 2014 work, Solar Reserve (Tonopah, Nevada) [Fig. 1] simulates the solar power project in Tonopah in Nevada, USA. More than ten thousand sun-tracking mirrors, called heliostats, focus the heat from the sun on a tower containing molten salt that generates electricity. The slow-moving camera simulation shows the solar reserve in sixty minutes from various angles. The work tracks the real-time movement of the sun mirroring the real-time sky of the original site in Nevada.

Using photographs as visual evidence of reality, Gerrard creates digital models from thousands of photographs of the place. With the change in medium from photographs to virtual models comes a change in the screen. From the screen of the camera, the photographs are transferred onto the screen of a computer for the virtual scene to be created. Heliostats are created as digital objects. Gerrard refers to such objects as "image objects" (Forrest 2015) due to their sharp resolution and immense detailing to appear real, not to forget that they are in fact based on real objects. This lends a sculptural quality to the objects, whereby they are created by hand but are mediated by a screen. These "sculptural photographs" (Groom 2010, 127), made on a screen, acquire a temporal character through the slow movement of camera and the movement of the sun. Gerrard's use of terms is testament to his artistic process that incorporates various media like photographs, sculpture, graphics, software and cinema. Additionally, this image-making process across media is facilitated by the synthesis of the multi-directional (on, through, in and behind - as illustrated by Mitchell [2015]) functions of screens.

The use of Unigine compels the consideration of the medium of gaming. Unigine is used to create 3-d and 2-d worlds and simulations mostly for video games. There are differences in the gaming world and the scene created by Gerrard that are not limited to using real places as a point of reference. The major difference lies in the engagement with the screen. Most virtual world games allow direct participation and influence on the virtual world. The screen 
is an instrument to enter the virtual space and explore the virtual landscape. Solar Reserve (Tonopah, Nevada) also creates a virtual world, but one that can only be observed from the outside and not manipulated by the audience. The large screen used during the display of the work does not encourage the haptic interaction and immersive potential of a gaming interface readily provided by Unigine. Instead, the large screen here reinforces the barrier between the viewing space and the virtual space onscreen. However, Gerrard insists on creating an experience in the "slippery" space between the real and its representation (Groom 2010, 131; Forrest 2015) through immense detailing of the scene and the real-time movement of the sun. Recognizing that the heliostats continue to track the sun even when the observer has left, makes the encounter with the screen at a particular time a memorable event. Moreover, during the making of the work, the images of the actual place in Tonopah are turned into data that is shared, edited and amplified via computer screens. This makes screens "information surfaces," a term used by Huhtamo (2004) to mark the transformation of screens with the changing social and cultural associations.

Gerrard's works have been referred to as post-cinematic (Becker 2014). A much discussed term in cinema studies, post-cinema broadly marks the changes in filmmaking, distribution and viewing modalities. Casetti uses the term "relocation of cinema" to describe the contemporary process that has repositioned the cinematic experience in new settings and new devices other than the cinema hall, such as computers and mobile phones (Casetti 2016). In this sense, Solar Reserve (Tonopah, Nevada) invokes post-cinematic experience and attention while viewing the simulation, as well as in the movement of cinema across different media during the making of the work, as it traverses across media from its photographic antecedents to the simulated camera movement, with the screen as its constant companion.

The screens are not merely mediating these media but performing the mutating tripartite relationship between the artwork, the artist and the observer, depending upon the screen modality. In this way, the intermediality of screens can be seen as a condition that operates in-between various components and practices. In their movement, the screens are unforming and reforming relationally with numerous entities. In this posturing they embody several processes taking place in the installation. The screens examine the intensities, enunciations and movements of the media art installation, whereby expressing the emergent properties of the installation. 


\section{b) Intermediality through in-betweenness}

Screens support the actions of the machinic components (hardware, software) and the humans in an interactive art installation by presenting the interface that communicates with the participant and the machine. The algorithmic functions, calculations and signal transfers made by software are concealed by the screens. On the other hand, screens express what is required and understood by the participant and leave out the processual information of the task. However, the concealment of the backend functions is not how the screens perform their gestural agency; instead, this lies in the capacity provided by in-betweenness.

Discussing interactive art, Munster $(2016,59)$ points out that "the inbetween is not an occupiable space but rather a dynamic and moving register of tendencies." Munster considers in-betweenness as a potential for deep and ever changing relations between technical entities and humans. This concept of in-betweenness can be applied to the idea of the gesturality of screens whereby it is able to form relations with various parts. Through the relations, the screens are able to execute certain functions, take on roles and express affects. This way betweenness is not a given position but is occupied depending on the arrangement of entities. The configuration of entities in turn depends upon their capacities of forming relations. In their relational entanglements, screens present new instances of interaction that may be used for further actions. Such an understanding of intermediality of screens highlights the transformations produced at a perceptive level as shifts in intensities and affects leading to a sensorial experience. These instances can be seen in media art installations where screens are generating medial interconnections.

Shadow 3 (2007) [Fig.2] by Shilpa Gupta is such an interactive media installation. In a dark room, the participants enter to face a large screen. The shadows of the participants are seen on the screen. However, these are not shadows but silhouettes that are captured by the motion capture device, Kinect, placed in front of the participants just below the screen. As the participants take their position in front of the screen, a string gets attached to their silhouettes on the screen. Shadows of numerous objects start sliding through the string towards the shadow of the audience members. The shadows of objects get attached to the silhouettes of the participants. The two are compiled into one assemblage. As the participants move, the attached objects move with them. Moreover, other participants can interact with these newly formed assemblages and transfer the objects from and to other participants. When the participant's silhouette is attached to the shadows of other objects, they become different objects. The fusion of the participants and the 
objects is a creation of temporary medial assemblages made in a particular time and space. They can be modified and reshaped by adding or lending shadows, that is, participants can extract objects from other people-object assemblages to add to their composition. During the transferring of the shadows of the objects, the screen facilitates two important connections: firstly, the combination of the shadows of the objects and the participants' bodies is different with each fusion, producing diverse shapes and affect; secondly, a collective experience is delivered in the assembling of the silhouettes of all participants at a given time on the screen.

In this installation, the screen is used by the participants to connect and interact with their silhouettes. It is an object of orientation through which they can engage and interact with their own body. Watching their silhouette on screen combining with various objects, the participants are able to experience their bodies external to themselves. The silhouette of the participant's body interacts with the shadows of objects that are not physically presented to the audience members. The screen offers a space where the participant's body can be transported and transfixed in a relationship with elements that have no actual form. Moreover, the participant's body evokes the outline of the body that we have become familiar with from devices such as Kinect and Wii that display the body as an onscreen avatar interacting with virtual objects. The screen here becomes a threefold space for display, interaction and performance.

A significant work that has used the silhouettes of human body on screen is VideoPlace (1985) by Myron Krueger. VideoPlace, a pioneering work in interactive media art, consists of several interactive programs. The silhouette of participants is projected on the screen in front of them. They can then interact with a variety of graphic images such as mountains, seas, miniature version of themselves, among others (Krueger 1985). There are a few works that use shadows on screen, such as Multiple Shadow House (2010) by Olafur Eliasson, Frost Frames (2000) by Shiro Takatani. But these are fundamentally different from VideoPlace and Shadow 3 as they rely on the shadows of the audience members and not their silhouettes. They require the audience to stand or move in front of the lights in the installation and cast shadows on a screen. The work Body Movies (2001) from the Relational Architecture series [Fig. 3] by Rafael Lozano-Hemmer also uses shadows of audience members. But the idea does not lie in casting shadows by obstructing light, instead, the shadows of the participants reveal other portraits that are projected on the screen. These other portraits are not seen in the brightly lit building, but can only be seen when the audience obstruct the light, cast their shadow and expose the portraits. 
In the three works, VideoPlace, Body Movies and Shadow 3, there is a connection that is formed not just with the images or shadows that are projected on the screen, but with the screen itself, though there are different modalities at work in these installations. All three works use silhouettes and shadows, so the visual details of the bodies are missing. While discussing VideoPlace, Munster $(2006,146)$ pointed out that since the silhouette onscreen is not the exact copy of the body in front of the screen, it is a "body of information" rather than one of "representation." It may seem that the gesturality of screens is the same in all three works, they are hiding and revealing, creating human-machine assemblages, relating different parts of the assemblage, and facilitating the interaction between machines and participants. However, the performance of that gesturality is different as each installation emphasizes a particular reconfiguration of relations among entities. The intermediality of screens grants a dynamic space in the varying qualities and configurations of the relations with other parts. In VideoPlace, the significant relationship lies in the gap that Munster $(2006,146)$ identified between the actual and information bodies. The gestural capacity of screens lies in maintaining this gap. The software provides instances that force the body to act in different ways. At the same time, the incapability of the software to replicate the participant's body means that the participant is constantly relating to the image on screen. In this way, screens facilitate the relation or the lack of it between the two kinds of bodies. In Body Movies, the screens are revealing the images that are already projected but not seen. As the images are seen only in the darkness of the shadow of the participants, the gesturality of screens is revealing the image.

The in-betweenness of screens makes it possible to circulate gesturality among the various entities of a set-up in such artworks. The intermediality of screens understood as an emergent state is then a mode of reconfiguring the relationship between image, artist, audience, and other components of the media art assemblage.

\section{References}

Acland, Charles R. 2012. The Crack in the Electric Window. Cinema Journal vol. 51, no. 2: 167-71.

Agamben, Giorgio. 2000 [1992]. Notes on Gesture. In Means without End. Notes on Politics, 49-60. Minneapolis: University of Minnesota Press.

Agamben, Giorgio. 2000 [1991]. Kommerell, or On Gesture. In Potentialities. Collected Essays in Philosophy, 77-85. Stanford: Stanford University Press. 
Balme, Christopher. 2004. Intermediality: Rethinking the Relationship between Theatre and Media. https://epub.ub.uni-muenchen.de/13098/1/Balme_13098. pdf. Last accessed 17. 03. 2019.

Becker, Carol. 2014. Here Comes the Sun. Art in America no. 24: https://www. artinamericamagazine.com/news-features/magazines/here-comes-the-sun/. Last accessed 17. 03. 2019.

Bennett, Jill. 2007. Aesthetics of Intermediality. Art History vol. 30, no. 3: 432-450.

Bennett, Jane. 2010. The Agency of Assemblages in Vibrant Matter. Durham, London: Duke University Press.

Bremner, Paul, Anthony G. Pipe, Chris Melhuish, Mike Fraser and Sriram Subramanian. 2011. The Effects of Robot-Performed Co-Verbal Gesture on Listener Behavior. Proc. 11th IEEE-RAS International Conference on Humanoid Robots11 (October): 458-465.

Bruno, Giuliana. 2014. Surface: Matters of Aesthetics, Materiality, and Media. China: The University of Chicago Press.

Cassetti, Francesco. 2016. The Relocation of Cinema. In Post-Cinema: Theorizing 21st-Century Film. Falmer: REFRAME Books. http://reframe.sussex.ac.uk/ post-cinema/introduction/. Last accessed 17. 03. 2019.

Chippendale, Paul. 2006. Towards automatic body language annotation. FGR '06 Proceedings of the 7th International Conference on Automatic Face and Gesture Recognition (April): 487-92.

Clüver, Claus. 2007. Intermedia and Interart Studies. In Changing Borders: Contemporary Positions in Intermediality, eds. Jens Arvidson, Mikael Askander, Jørgen Bruhn and Heidrun Führer. Intermedia Studies Press.

Darwin, Charles. 1965 [1872]. The Expression of Emotions in Man and Animals. Chicago: University of Chicago Press.

Deleuze, Gilles and Felix Guattari. 2017 [1980]. A Thousand Plateaus: Capitalism and Schizophrenia. Chennai, India: Bloomsbury.

Eibl-Eibesfeldt, Irenäus. 1972. Similarities and Differences between Cultures in Expressive Movements. In Non-verbal Communication, ed. R. A. Hinde, 297314. London: Cambridge University Press.

Elleström, Lars. 2010. The Modalities of Media: A Model or Understanding Intermedial Relationship. In Media Borders, Multimodality and Intermediality, ed. Lars Elleström, 11-48. London: Palgrave Macmillan.

Forrest, Nicholas. 2015. Interview: John Gerrard on his "Slippery" Sims at Thomas Dane Gallery. In Blouin Artinfo, February 16. http://uk.blouinartinfo. 
com/news/story/1103413/interview-john-gerrard-on-his-slippery-sims-atthomas-dane. Last accessed 17. 03. 2019.

Friedberg, Anne. 2006. The Virtual Window: From Alberti to Microsoft. Spain: MIT Press.

Groom, Simon. 2010. John Gerrard and Simon Groom in conversation at the Scottish National Gallery of Modern Art. In John Gerrard. Madrid: IvoryPress. Grusin, Richard. 2010. Premediation: Affect and Mediality after 9/11. New York: Palgrave Macmillan.

Gunning, Tom. 2006 [1986]. The Cinema of Attraction[s]: Early Film, Its Spectator and the Avant-Garde. In Cinema of Attractions Reloaded, ed. Wanda Strauven, 381-88. Amsterdam: Amsterdam University Press.

Higgins, Dick. 2001 [1981]. Intermedia. Leonardo vol. 34, no. 1 (February): 49-54. Huang, Chien-Ming and Bilge Mutlu. 2013. Modeling and Evaluating Narrative Gestures for Humanlike Robots. In Proceedings of the 2013 Robotics: Science and Systems Conference.

(RSS'13). http://www.roboticsproceedings.org/rss09/p26.pdf. Last accessed 19. 07. 2019.

Huhtamo, Erkki. 2012. Screen Tests: Why Do We Need an Archaeology of the Screen? Cinema Journal vol. 51, no. 2: 144-48.

Jenkins, Henry. 2006. Convergence Culture. Where Old and New Media Collide. New York, London: New York University Press.

Krueger, Myron W., Thomas Gionfriddo and Katrin Hinrichsen. 1985. VIDEOPLACE-an artificial reality. ACM SIGCHI Bulletin vol. 16, no. 4: 35-40. LaFrance, Marianne and Clara Mayo. 1978. Cultural Aspects of Nonverbal Communication. International Journal of Intercultural Relations vol. 2, no. 1 (Spring): 71-89.

Langford, Michelle. 2006. Allegorical Images: Tableau, Time and Gesture in the Cinema of Werner Schroeter. Bristol: Intellect.

Laurel, Brenda. 1991. Computer as Theatre. Reading, Mass.: Addison-Wesley Publications.

Lindsay, Vachel. 1915. The Art of the Moving Picture. New York: Macmillan. Lippit, Akira Mizuta. 2008. Digesture: Gesture and Inscription in Experimental Cinema. In Migrations of Gesture, eds. Carrie Noland and Sally Ann Ness, 113132. Minneapolis: University of Minnesota Press. 
Lord, Susan, and Janine Marchessault, eds. 2007. Fluid Screens, Expanded Cinema. Toronto: University of Toronto Press.

Manovich, Lev. 1998. Towards an Archaeology of the Computer Screen. In Cain, Abel or Cable? The Screen Arts in the Digital Age, eds. Thomas Elsaesser and Kay Hoffmann, 27-43. Amsterdam: Amsterdam University Press.

Massumi, Brian. 2002. Parables of the Virtual. Durham, NC: Duke University Press.

Metz, Christian. 1982. Psychoanalysis and Cinema: The Imaginary Signifier. London: Macmillan.

Mitchell, W. J. T. 2015. Screening nature (and the nature of the screen). New Review of Film and Television Studies vol. 13, no. 3: 231-46.

Mondloch, Kate. 2010. Screens: Viewing Media Installation Art. Minneapolis : University of Minnesota Press.

Müller, Jürgen E. 2010. Intermediality and Media Historiography in the Digital Era. Acta Universitatis Sapientiae: Film And Media Studies vol. 2: 15-38 .

Mulvey, Laura. 1999 [1975]. Visual Pleasure and Narrative Cinema. In Film Theory and Criticism: Introductory Readings, eds. Leo Braudy and Marshall Cohen, 833-44. New York: Oxford UP.

Munster, Anna. 2006. Materializing New Media: Embodiment in Information Aesthetics. Lebanon, USA: Dartmouth College Press.

Munster, Anna. 2016. Into 'Inter': the Between in Interacting. Rivista di estetica vol. 63: 56-67.

Noland, Carrie. 2008. Introduction. In Migrations of Gesture, eds. Carrie Noland and Sally Ann Ness, ix-xxviii. Minneapolis: University of Minnesota Press.

Paech, Joachim. 2014. Intermediate Images. Acta Universitatis Sapientiae: Film And Media Studies vol. 9: 31-49.

Paul, Christiane. 2015. Mediations of Light: Screens as Information Surfaces. In Digital Light, eds. Sean Cubitt, Daniel Palmer and Nathaniel Tkacz, 179-92. London: Open Humanities Press.

Pethő, Ágnes. 2014. The Garden of Intermedial Delights. Screen vol. 55, no. 4: 471-489.

Pisters, Patricia. 2015. Image as Gesture: Notes on Aernout Mik's Communitas and the Modern Political Film. Journal for Cultural Research vol. 19, no. 1: 69-81.

Rajewsky, Irina. 2005. Intermediality, Intertextuality, and Remediation. Intermédialités no. 6 (Autumn): 43-64.

Shaw, Jeffrey and Peter Weibel, eds. 2003. Future Cinema: The Cinematic Imaginary after Film. Cambridge, Mass.; London, England: MIT Press. 
Spielmann, Yvonne. 2001. Intermedia in Electronic Images. Leonardo vol. 34, no. 1: 55-61.

Stern, Lesley. 2008. Ghosting: The Performance and Migration of Cinematic Gesture, Focusing on Hou Hsiao-Hsien's Good Men, Good Women. In Migrations of Gesture, eds. Carrie Noland and Sally Ann Ness, 185-216. Minneapolis: University of Minnesota Press.

Trodd, Tamara, ed. 2011. Screen/Space. The Projected Image in Contemporary Art. Manchester: Manchester University Press.

Verhoeff, Nanna. 2012. Mobile Screens: The Visual Regime of Navigation. Amsterdam: Amsterdam University Press.

Youngblood, Gene. 1970. Expanded Cinema. New York: Dutton.

Zapp, Andrea and Martin Rieser, eds. 2002. New Screen Media: Cinema/Art/ Narrative. London, Karlsruhe: British Film Institute and Zentrum für Kunst und Medientechnologie Karlsruhe.

\section{List of Figures}

Figure 1. Installation shot of Solar Reserve (Tonopah Nevada) by John Gerrard on a $28 \mathrm{ft} \mathrm{X} 24 \mathrm{ft} \mathrm{LED} \mathrm{display} \mathrm{screen} \mathrm{at} \mathrm{Lincoln} \mathrm{Centre,} \mathrm{New} \mathrm{York,} \mathrm{USA,} 2014$. Photographer: Iñaki Vinaixa. Courtesy: The Artist, Thomas Dane Gallery, London and Simon Preston Gallery, NY.

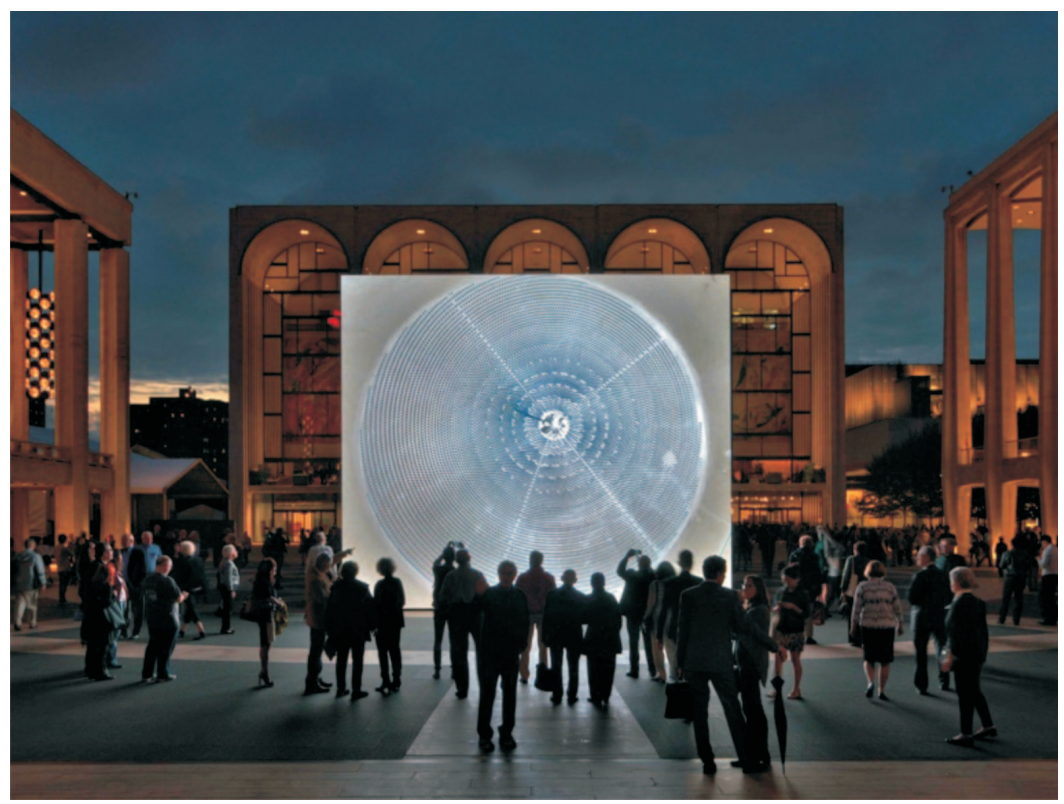


Figure 2. Shilpa Gupta, Untitled (Shadow 3) 2007. Interactive video projection incorporating the viewer's simulated shadow. Photographer: Anil Rane. Courtesy: The Artist \& Galleria Continua / Le Moulin, San Gimignano / Beijing / Les Moulins / Habana.



Figure 3. Installation shot of Body Movies, Relational Architecture by Rafael Lozano-Hemmer at Schouwburgplein, V2 Cultural Capital of Europe, Rotterdam, The Netherlands. Rafael Lozano-Hemmer, Body Movies, Relational Architecture 6. Interactive projection, 2001. Photographer: Jan Sprij. Courtesy: Rafael LozanoHemmer, Antimodular Inc.

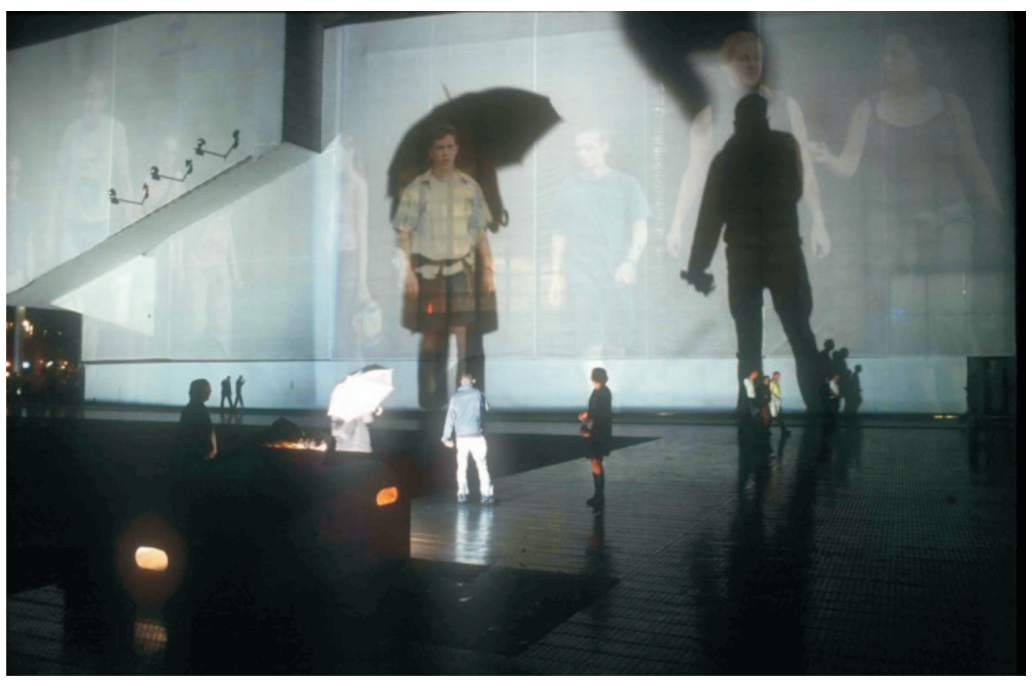

